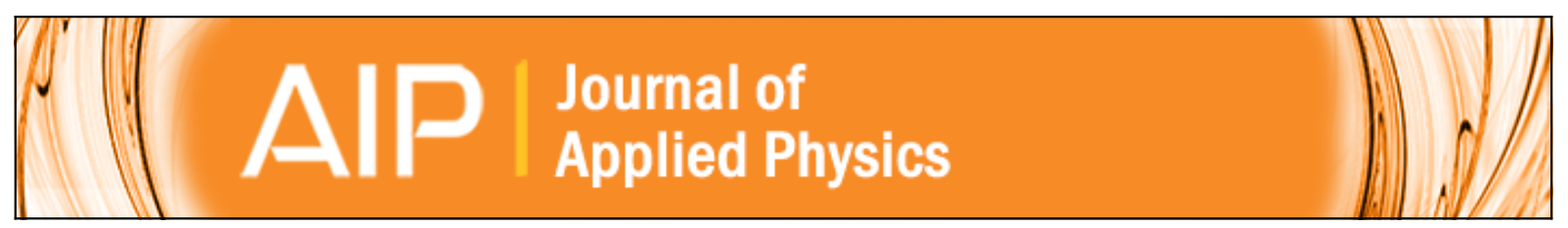

\title{
Absorption spectroscopy of isolated magnetic antivortices
}

Matthias Pues, Michael Martens, and Guido Meier

Citation: Journal of Applied Physics 116, 153903 (2014); doi: 10.1063/1.4897648

View online: http://dx.doi.org/10.1063/1.4897648

View Table of Contents: http://scitation.aip.org/content/aip/journal/jap/116/15?ver=pdfcov

Published by the AIP Publishing

\section{Articles you may be interested in}

High-frequency switching of magnetic bistability in an asymmetric double disk nanostructure

Appl. Phys. Lett. 104, 112405 (2014); 10.1063/1.4869024

Reliable nucleation of isolated magnetic antivortices

Appl. Phys. Lett. 100, 162404 (2012); 10.1063/1.3698150

Energy surface model and dynamic switching under alternating field at microwave frequency

Appl. Phys. Lett. 94, 102506 (2009); 10.1063/1.3097229

Current- and field-driven magnetic antivortices for nonvolatile data storage

Appl. Phys. Lett. 94, 062504 (2009); 10.1063/1.3072342

Vortex motion in chilarity-controlled pair of magnetic disks

Appl. Phys. Lett. 90, 132501 (2007); 10.1063/1.2716861

You don't

still use this

cell phone

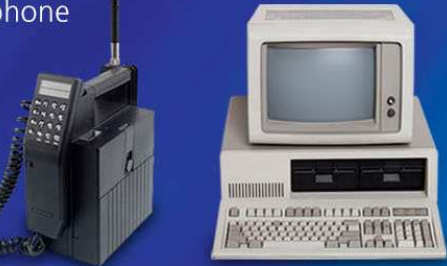

Why are you still using an AFM designed in the 80 's?
It is time to upgrade your AFM

Minimum $\$ 20,000$ trade-in discount for purchases before August 31st

Asylum Research is today's technology leader in AFM 


\title{
Absorption spectroscopy of isolated magnetic antivortices
}

\author{
Matthias Pues, ${ }^{1, a)}$ Michael Martens, ${ }^{1}$ and Guido Meier ${ }^{1,2}$ \\ ${ }^{1}$ Institut für Angewandte Physik und Zentrum für Mikrostrukturforschung, Universität Hamburg, Jungiusstraße \\ 11, 20355 Hamburg, Germany \\ ${ }^{2}$ Max Planck Institute for the Structure and Dynamics of Matter, Luruper Chaussee 149, 22761 Hamburg, \\ Germany
}

(Received 27 August 2014; accepted 30 September 2014; published online 15 October 2014)

\begin{abstract}
We present an analysis of the dynamics of isolated magnetic antivortices preformed by high frequency absorption measurements from the linear via the non-linear to the switching regime. Static magnetic bias fields are used to deflect the antivortex out of the equilibrium position and the shift of the resonance frequency of the gyrotropic eigenmode is observed. The results from the absorption measurements for highly anisotropic annihilation fields of the antivortex are compared with magneto-resistance measurements and micromagnetic simulations. (C) 2014 AIP Publishing LLC. [http://dx.doi.org/10.1063/1.4897648]
\end{abstract}

\section{INTRODUCTION}

Isolated vortices and antivortices have been studied as potential candidates for fast non-volatile data-storage. ${ }^{1-3}$ These magnetic singularities can be found in thin-films of soft ferromagnetic materials like permalloy $\left(\mathrm{Ni}_{80} \mathrm{Fe}_{20}\right)$. The singularities are characterized by a special curling of the inplane magnetization around a small core region, where the magnetization tilts out-of-plane, either up or down. This so called polarization $p= \pm 1$ can be utilized to store a bit of information.

In order to switch the polarization, several different mechanisms for both vortices and antivortices have been described. The gyrotropic eigenmode of the vortex or antivortex, a circular motion of the core around the equilibrium position, can be excited by oscillating external fields ${ }^{4-7}$ or spin currents ${ }^{8,9}$ either in a continuous or pulsed fashion. ${ }^{10,11}$ Once the core reaches a critical velocity, the radius of the trajectory does no longer increase, but the magnetization radially inwards of the core is deformed and a vortexantivortex pair with an opposite polarity is created. The original core annihilates with its counterpart. In case of a vortex, it annihilates with the antivortex of the pair, and a single vortex with opposite polarity remains. A corresponding process for antivortices exists. ${ }^{4,5,12,13}$ Since both singularities are involved in either switching process, a detailed knowledge of the dynamics of vortices and antivortices is needed for a complete understanding of the involved processes.

Vortices have been studies intensively, since they can be found as the ground state in thin-film structures with proper thickness and width that resemble a disc ${ }^{14,15}$ closing the magnetic flux. In contrast to this, experimental studies on antivortices are more complicated as their magnetic structure is characterized by four alternating poles giving rise to a complex shape in which the antivortex can be stabilized. ${ }^{7,9,16,17}$ In these thin-film structures, the antivortex is, although stable, not easily recoverable once destroyed and mostly found as an as-grown state. Our method presented in

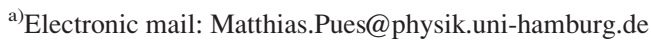

Ref. 17 for a reliable antivortex nucleation in a specially shaped structure enables an analysis of the antivortex dynamics by means of high frequency (hf) absorption measurements as well as magneto resistance (MR) measurements for the static behavior of isolated antivortices.

\section{SAMPLES AND MEASUREMENT TECHNIQUES}

Samples are fabricated by electron-beam lithography using a Zeiss Supra 55 SEM with a Raith lithography system and lift-off processing on silicon substrates. For absorption measurements, $85 \varphi$-shaped permalloy $\left(\mathrm{Ni}_{80} \mathrm{Fe}_{20}\right)$ elements with a thickness of $50 \mathrm{~nm}$ and a wire width $w$ of $1.1 \mu \mathrm{m}$ are deposited by thermal evaporation as well as the overlaying $80 \mathrm{~nm}$ thick copper stripline, capped by $5 \mathrm{~nm}$ gold, see Fig. 1. For resistance measurements, a single permalloy element is contacted by dc-magnetron sputtered gold leads. Before deposition of the gold layer, the contact surface is

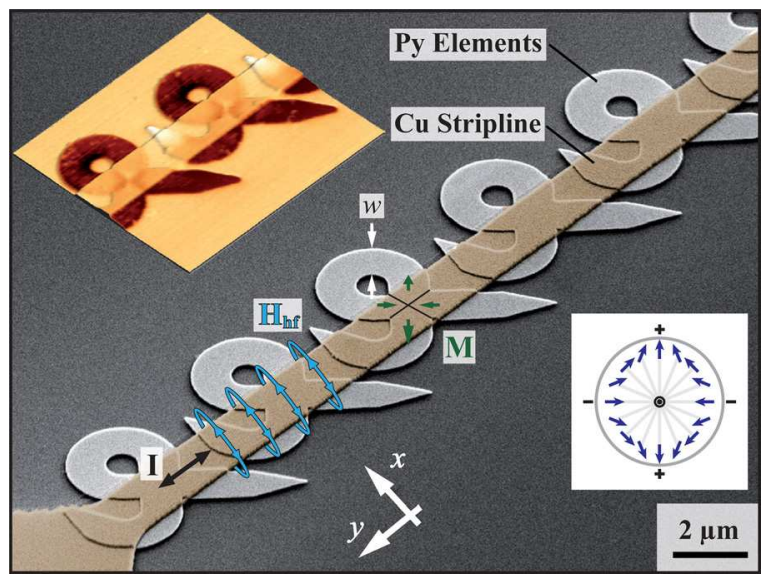

FIG. 1. Scanning-electron micrograph of several $\varphi$-shaped permalloy microstructures overlaid by a copper stripline for high-frequency absorption measurements. The surrounding Oersted-field of the high-frequency current is schematically depicted. The inset in the upper left shows a magnetic force micrograph with antivortices in each element, where the three-dimensional depiction of the topography is overlaid by the color coded magnetic information. ${ }^{17,18}$ The inset in the lower right shows the in-plane magnetization vector field of an antivortex. 
cleaned in situ via radio frequency argon-plasma etching to ensure a good electrical contact and a $3 \mathrm{~nm}$ adhesive aluminum layer is deposited without breaking the vacuum.

The absorption spectroscopy is performed in a serial setup of an Agilent Technologies E8257D analog signal generator, the sample, and an Agilent E4418B power meter with an Agilent E9304A sensor. ${ }^{6}$ The electrical connection from SMA connectors on a printed circuit board to the broadened ends of the copper stripline is realized by aluminum wire bonding. The ground signals are closed on the printed circuit board, since no impedance matched ground-signal-ground layout is necessary within the frequency range used here. A two-dimensional electromagnet with two pairs of pole pieces surrounding the sample, that is controlled via Hall sensor feedback, ensures the precise remanence free control of the external in-plane field necessary for the generation of the antivortices. ${ }^{17}$ The gyrotropic mode of the antivortices is excited harmonically by the unidirectional alternating Oersted field of the stripline. At resonance, the antivortices gyrate with a maximum radius around their equilibrium position. The power absorption of the antivortex ensemble results in an increase of the total stripline impedance. This conversion has been discussed in Ref. 6. In order to detect the small signal $\Delta R$ caused by the antivortex ensemble compared to the resistance of the stripline $\left(R_{\mathrm{SL}} \approx 70 \Omega\right)$, a reference signal has to be measured. This is done by saturating the magnetization of all structures at $90 \mathrm{mT}$ in $x$-direction and setting the field back to the bias field used to deflect the antivortex from its equilibrium position. Note, that for some experiments, the bias field can be zero. Application of the saturation field ensures that a homogeneous magnetization is present in the wire junctions of the structures. Antivortices are then generated by a two dimensional field sequence that has been presented in our previous work, see Ref. 17, and the signal difference of both magnetic states is determined. Thus for every frequency step, the antivortices are newly generated. This method is prone against temperature or possible other drifts of the resistance of the whole setup. Moreover, possible magnetic state changes induced by the bias field have no effect on the following data points. Since the method used to generate the antivortices also gives control over their orientation, antivortices with $c=-1$ are investigated in all measurements presented here. The measurements are performed at $T=21.5^{\circ} \mathrm{C}$ controlled with a precision of $0.15 \mathrm{~K}$.

We have performed complementary magneto resistance measurements in lock-in technique. Figure 2(a) depicts a microstructure with Au contacts. The antivortex state is investigated by comparison of the resistance in saturation parallel to the current flow direction, indicated in Fig. 2(a), and the resistance after antivortex generation and application of the bias in-plane field with angle $\Theta$ for each data point. The magnetization pattern of the antivortex approximately consists of four triangularly shaped uniformly magnetized domains, see Figs. 2(b) and 2(c). For an estimation of the MR signal of the antivortex state, the ratio of the domain size parallel and perpendicular to the current flow is constant for a core deflection in an external field. When we assume a homogeneous current flow between the contact leads, a
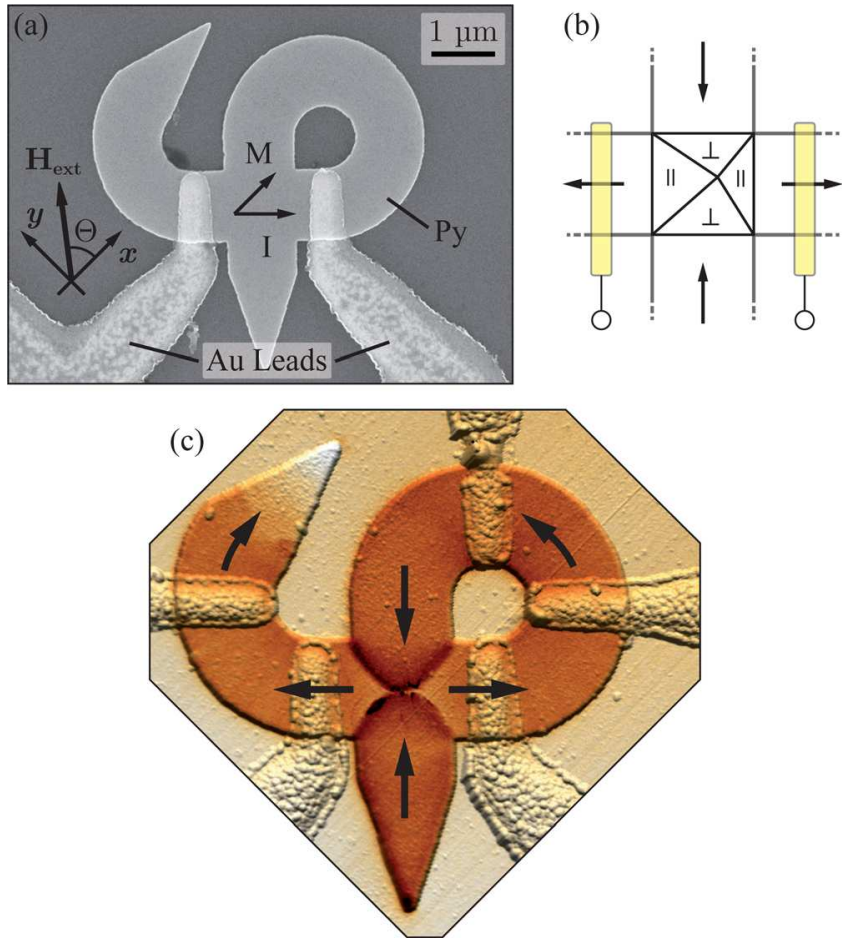

FIG. 2. (a) Scanning electron micrograph of a $\varphi$-shaped structure with $\mathrm{Au}$ contact leads for resistance measurements. (b) Scheme of a deflected antivortex. The triangularly shaped domains are denoted as parallel or perpendicular to the current flow. (c) Combined atomic and magnetic force micrograph of a contacted $\varphi$-structure containing an antivortex with an orientation of $c=-1$. For the MR measurements presented here, the structure with two contacts shown in (a) is used.

constant magnetization within the wire arms, and no deformation of the $90^{\circ}$ domain walls, the MR signal is expected to be constant to a good approximation.

\section{RESULTS AND DISCUSSION}

The resonance frequency of the gyrotropic eigenmode of isolated antivortices is determined by means of absorption spectroscopy. Figure 3(b) shows a typical absorption spectrum for the antivortex ensemble described above with a resonance frequency of $f_{\text {res }}=(169.4 \pm 0.3) \mathrm{MHz}$. This frequency is approximately $40 \%$ lower than the resonance frequency of a vortex confined in a square-shaped element with the same film thickness, and edge length comparable to the wire width. ${ }^{6}$ The lower frequency indicates a weaker confining potential if the antivortex is considered as a rigid quasiparticle as it is successfully done for vortices.

A variation of the excitation power and with that the exciting Oersted field ${ }^{21}$ is depicted in Fig. 3(a). ${ }^{22}$ For low excitation powers, the resonance frequency is rather constant. In this regime (1), the gyration of the antivortex can be described as a rigid quasiparticle in a nearly parabolic potential. ${ }^{20}$ If the excitation power is increased, the resonance frequency drops significantly about $20 \%$ in regime (2). In addition to the frequency drop, an asymmetric absorption curve can be observed in this non-linear regime, see Fig. 3 (c). This asymmetry has also been reported for numerical simulations and measurements of resonance curves of vortices by Drews et al. in Ref. 19. The core switching regime (3) 

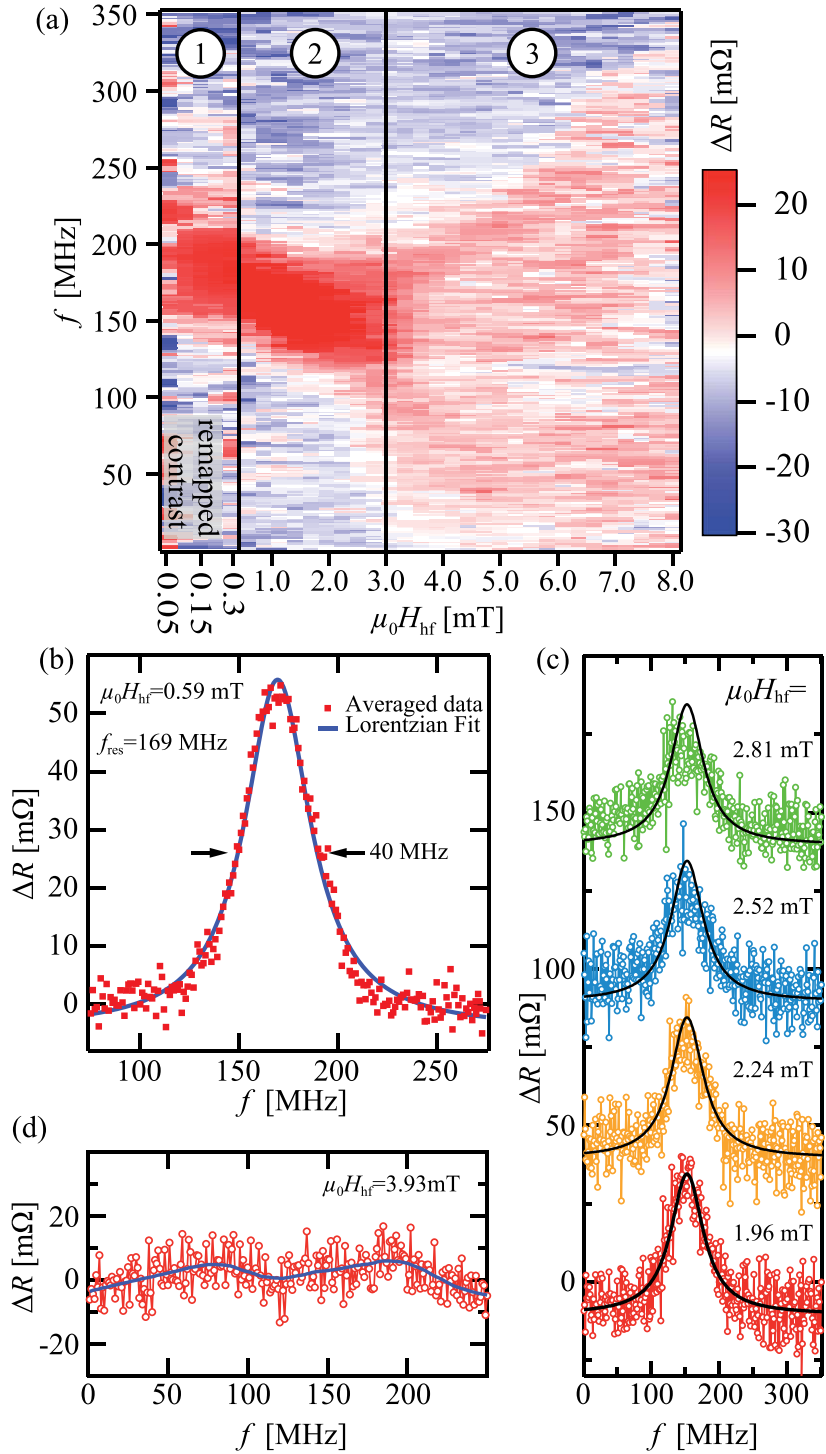

FIG. 3. (a) Influence of the exciting field strength $H_{\mathrm{hf}}$ on the absorption spectrum. Three regions can be distinguished: (1) linear gyrotropic motion, (2) non-linear gyrotropic motion, and (3) continuous switching of the polarity of the antivortices. In regime (3), the cone shaped absorption signal is caused by continuous switching processes. Note the different field step sizes in region (1) of about $0.05 \mathrm{mT}$ compared to regions (2) and (3) with $0.28 \mathrm{mT}$. (b) Average of 17 frequency sweeps in the linear regime. The absorption signal shows a Lorentzian shape caused by the resonant excitation of the gyrotropic eigenmode of the antivortex ensemble. (c) Absorption signals for an increasing excitation fields in the non-linear regime of the gyrotropic mode. The curves are offset successively by $50 \mathrm{~m} \Omega$. In order to show the increasing asymmetry and red shift of the absorption curves, ${ }^{19,20}$ the same Lorentzian fit at the lowest excitation field strength of $1.96 \mathrm{mT}$ is plotted as a solid line for all data curves. (d) Absorption signal from the core switching regime. The solid line is a guide to the eye.

is reached at $\mu_{0} H_{\mathrm{hf}}=3 \mathrm{mT}$, indicated by the characteristic cone shaped signal $^{23}$ above $3 \mathrm{mT}$. The Oersted field to induce antivortex core switching is about six times the field strength needed to induce core switching in a similar setup for vortices. ${ }^{6,23}$ The continuous switching of the polarity of the core is possible, since the linear excitation by the stripline can couple to both the clockwise and the counterclockwise eigenmodes of the antivortex, in contrast to an excitation by rotational Oersted fields ${ }^{9}$ or rotational spin currents. $^{7}$
The absorption signal is reduced for strong excitation fields above $3 \mathrm{mT}$ near the resonance frequency, which can be explained by the instability of the antivortex state, see Fig. 3(d). If the antivortex switches its core polarization multiple times at high gyration radii, there could be a probability that it moves away from the equilibrium position in the middle of the wire junction into one of the arms and is destroyed there. Since the absorption measurement is a time integrating method, this process does not contribute to the ensemble signal anymore. For vortices especially in discs, these considerations are irrelevant, since the vortex state is the energetically favored state. Consequently, even if a vortex is destroyed by a strong excitation, it will recover shortly after and will again contribute to the absorption signal.

To probe the confining potential of the antivortex, a static in-plane field is applied in eight different directions, $H_{\text {ext }}(\Theta)$, in order to deflect the antivortex from the center of the wire junction. Since the generation process of the antivortices determines the orientation of all antivortices in the ensemble, here $c=-1$, a single deflection direction of the whole ensemble can be ensured. A detailed description of the shift of the energy minimum due to this Zeeman field for vortices is presented in Ref. 24. For the absorption spectroscopy, see Fig. 4(a), a low excitation field in the harmonic regime of $\mu_{0} H_{\mathrm{hf}}=0.3 \mathrm{mT}$ is chosen. The resonance frequency dependence of the static bias field $H_{\text {ext }}(\Theta)$ shows a different behavior for different field angles as well as a varying annihilation field $H_{\text {an }}(\Theta)$ of the antivortices. At the annihilation field, the antivortex is pushed out of the wire junction and consequently, the absorption signal vanishes. Three types of frequency shifts can be distinguished. The frequency shift labeled (I) exhibits a small drop in the resonance frequency of $6 \mathrm{MHz}$, which corresponds to about $4 \%$, as well as a decrease in the absorption. The annihilation field is around $2 \mathrm{mT}$. The second type (II) is characterized by a similarly small annihilation field, but shows a frequency increase of about $33 \mathrm{MHz}(20 \%)$ with an increase of the absorption signal for bias field values close to the annihilation field. The third type (III) has relatively high annihilation fields up to $8 \mathrm{mT}$ and the resonance frequency rises about $25 \mathrm{MHz}$ $(15 \%)$. The small annihilation fields of antivortices in comparison to annihilation fields of vortices of about $35 \mathrm{mT}$ (Ref. 6) indicate again the comparably very shallow potential, which confines the antivortex within the wire junction. Moreover, the anisotropy of the annihilation fields and the frequency shifts shows a strong influence of the wire arms and the asymmetry of the structure on the antivortex.

To compare the drastic asymmetry of the annihilation field for gyrating antivortices with the annihilation fields for a static antivortex, magneto-resistance measurements are performed, see Fig. 4(b). The annihilation fields are indicated by abrupt resistance changes closest to zero fields in the MR signal. The MR signal jumps from a nearly constant plateau, as expected for the deflected antivortex state, to a MR signal indicating a magnetization diagonal, perpendicular, or parallel to the current flow, see insets in Fig. 4(b). For some field angles, the annihilation of the antivortex is followed by additional step-like transitions most likely due to sudden depinning processes of domain walls from the corners of the 
(a)

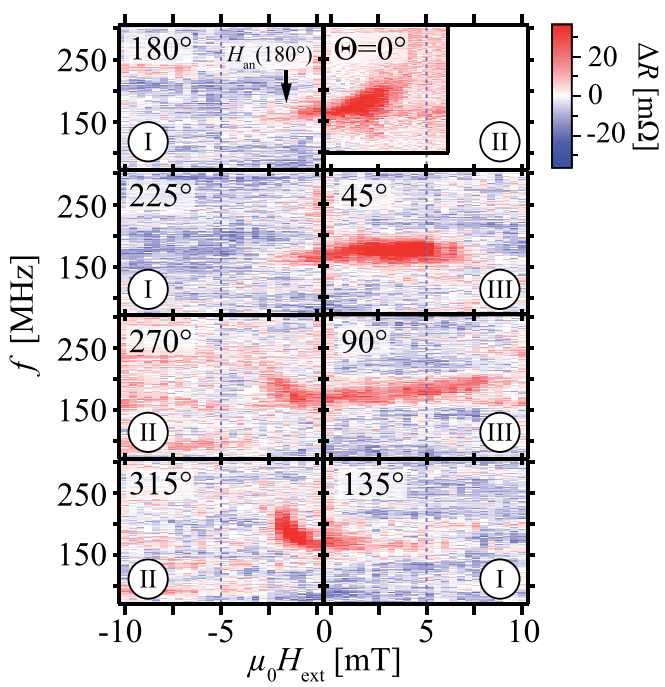

(b)

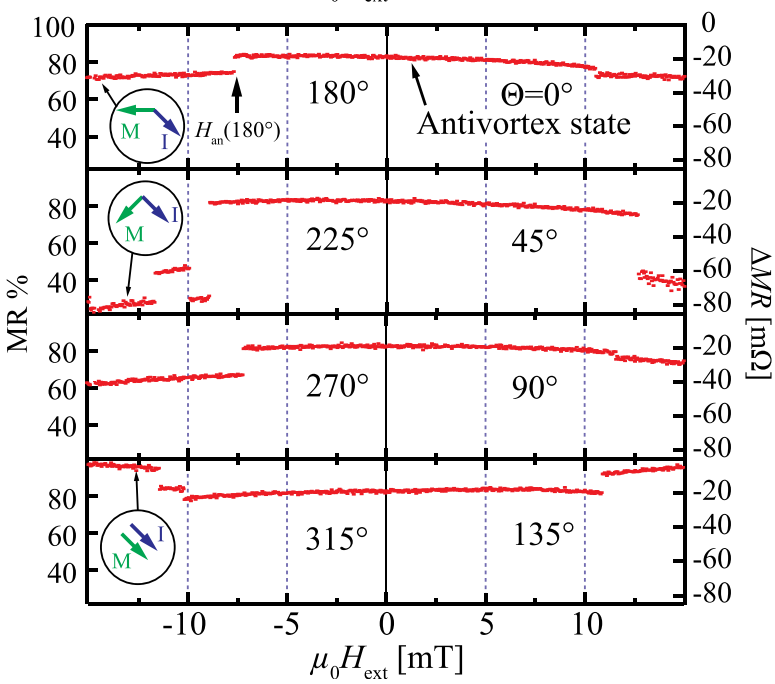

FIG. 4. (a) Dependence of the antivortex resonance on a static bias field $H_{\text {ext }}(\Theta)$. (b) Magneto resistance of the wire junction of a $\varphi$-shaped microstructure that contains an antivortex at zero field. The annihilation fields of the antivortex vary strongly between the two experiments. The excited antivortex is destroyed at lower static field strengths. In the absorption spectra, three types (I, II, and III) of frequency shifts can be distinguished.

junction. As every data point represents a whole field sweep starting after an antivortex nucleation from zero field to either positive or negative field values, the smooth field dependence of the MR signal for each field angle indicates both a successful antivortex nucleation for every data point and a single path for the expulsion of the antivortex. The annihilation fields range from $7.2 \mathrm{mT}$ to $12.7 \mathrm{mT}$ and show a similar but much less distinct asymmetry as those derived from the absorption measurements, see Fig. 5(a).

Analogous to the MR measurements, micromagnetic simulations are performed with the OOMMF code ${ }^{25}$ where the deflection of the antivortex by a quasi static in-plane field $H_{\text {ext }}(\Theta)$ for eight different field angles is investigated. The simulated element is mapped from a scanning electron micrograph of a real element, ensuring the same dimensions and edge roughness. Typical material parameters for permalloy are used, a saturation magnetization of $M_{\mathrm{S}}=8 \times 10^{5} \mathrm{~A} \mathrm{~m}^{-1}$, and an exchange constant of $A=1.3 \times 10^{-11} \mathrm{~J} \mathrm{~m}^{-1}$. An artificially high Gilbert damping of $\alpha=0.5$ is used, as only the (a)

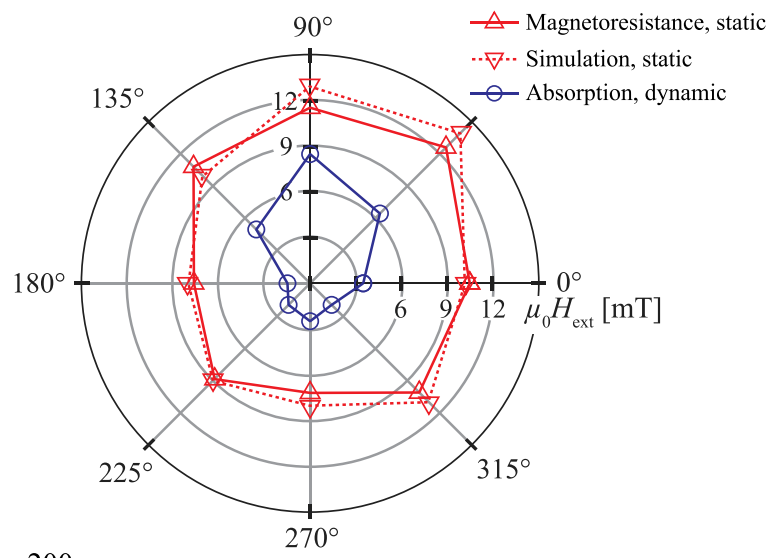

(b)

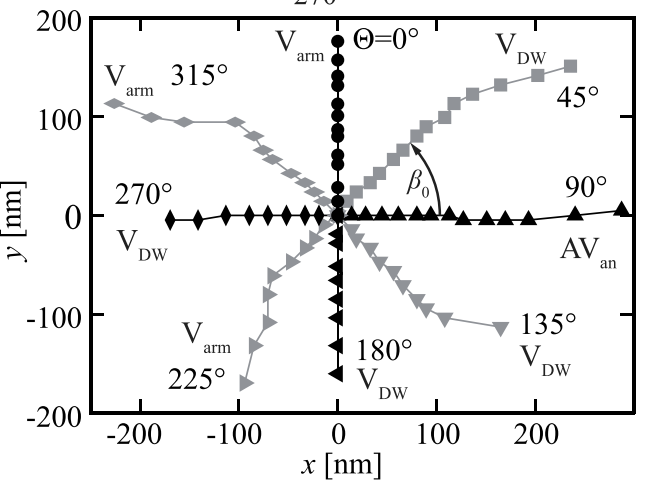

(c)

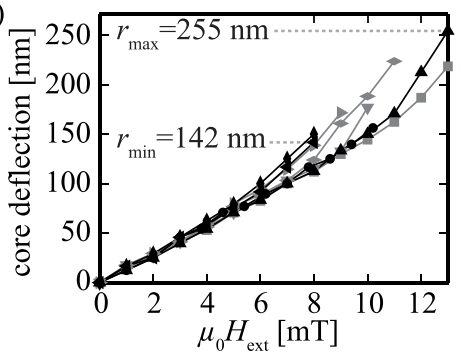

(d)

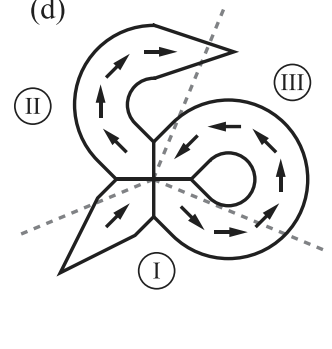

FIG. 5. (a) Comparison of the annihilation fields of the deflected antivortex for different in-plane field angles of the static field $H_{\text {ext }}$. Annihilation fields from magneto-resistance measurements, micromagnetic simulations, and high frequency absorption measurements are shown. (b) Simulated deflection from the equilibrium position of the core for bias fields with the indicated angle. The different annihilation processes $\left(V_{\mathrm{arm}}, \mathrm{V}_{\mathrm{DW}}\right.$, and $\left.A \mathrm{~V}_{\mathrm{an}}\right)$ for each angle are explained in the text. (c) Distance from the equilibrium position at zero field of the antivortex core depending on the bias field obtained by micromagnetic simulations. (d) Sketch of a $\varphi$-shaped structure. A deflection of the antivortex into one of the marked regions corresponds to the resonance frequency shift types from Fig. 4(a).

new equilibrium position of the antivortex for the given bias field is of interest here. Using a realistic damping value, the step-like increase of the field would result in an unrealistic spin-wave generation. The cell size of the simulation mesh is chosen to be $5 \times 5 \times 25 \mathrm{~nm}^{3}$.

The annihilation fields derived from these simulations match the ones determined by the MR measurements, see Fig. 5(a). Furthermore, the simulations give a deep insight in the deflection behavior of the antivortex, as well as in the annihilation process for different field directions. Figure 5(c) shows the core deflection distance from its equilibrium position for all fields with a minimal deflection of $r_{\min }=142 \mathrm{~nm}$ and a maximal deflection of $r_{\max }=255 \mathrm{~nm}$. A linear dependence of the core deflection on the external field with a rate of $(14.6 \pm 0.7) \mathrm{nm} / \mathrm{mT}$ can be found up to about $5 \mathrm{mT}$, 
indicating the parabolic confining potential. For higher field strengths, the core deflection rate increases up to the annihilation of the antivortex. This increase shows that the confining potential becomes shallower once the antivortex is pushed into the arms, which is contrary to the behavior of isolated vortices. A vortex is confined in a closed microstructure like a disc or a square, resulting in a deviation from the parabolic confining potential near the boundary of the structure. Thus, a vortex needs much stronger fields to be pushed towards the boundaries of the structure. ${ }^{19,24,26}$ In the graph showing the core position for all field strengths and angles, see Fig. 5(b), another effect of the open junction can be seen. For field strengths causing a linear deflection rate up to $5 \mathrm{mT}$, the displacement of the core follows a certain angle. This azimuth angle $\beta_{0}$ to the new equilibrium position of the core, see Fig. 5(b), can be generally derived by $\beta_{0}=n\left(\Theta+\pi-\Phi_{0}\right)=n(\Theta+\pi-c \pi / 2)$ taking a rotational symmetric potential and the Zeeman energy into account. Here, the antivortex with a winding number ${ }^{27}$ of $n=-1$ and the fixed orientation of $c=-1$ yields $\beta_{0}=-\Theta-3 \pi / 2$. At certain field strengths, the core deflection deviates from this direction, mostly for the deflection into the arms. This can be attributed to the depinning of the $90^{\circ}$ domain walls from the corners of the wire junction, see Fig. 6(d), and a deformation of the walls. Moreover, for high field strengths the magnetization within the arms whose magnetization points antiparallel to the external field starts to bend in a zigzag fashion, leading to the deflection of the core away from the center of the arm. This behavior is not described by a simple rigid quasiparticle model of the antivortex, where the antivortex is confined in a parabolic potential. An extension of the parabolic potential to describe the deviation of the potential towards the boundaries of the structure has been done for vortices in squares ${ }^{24}$ but for antivortices this approach poses several difficulties. For antivortices in $\varphi$-shaped structures, the potential is highly anisotropic, whereas for vortices in squares only two different field angles, towards the edge of the square or diagonally have to be considered.

Similar shifts of the resonance frequency of the antivortex can be found for adjacent field angles and considering the deflection angle of the antivortex core caused by the external field reveals the influence of the $\varphi$-shaped structure onto the confining potential, see Fig. 5(d), even though the wire junction in which the antivortex is confined is completely rectangular. Thus, a much more complex potential confines the antivortex.

An analysis of the antivortex annihilation for each field angle $\Theta$ reveals three different processes, as indicated in Fig. 5(b). In Fig. 6, two of these annihilation processes are examplarily shown in the micromagnetic simulations for field angles of $\Theta=0^{\circ}$ in Figs. 6(a)-6(c), and $90^{\circ}$ in Figs. 6(d)-6(f). At an external field of $\mu_{0} H_{\text {ext }}\left(0^{\circ}\right)=11 \mathrm{mT}$, the magnetization in the upper right curved wire folds, forming a $180^{\circ}$ domain wall, see Fig. 6(a). From this wall, a vortex

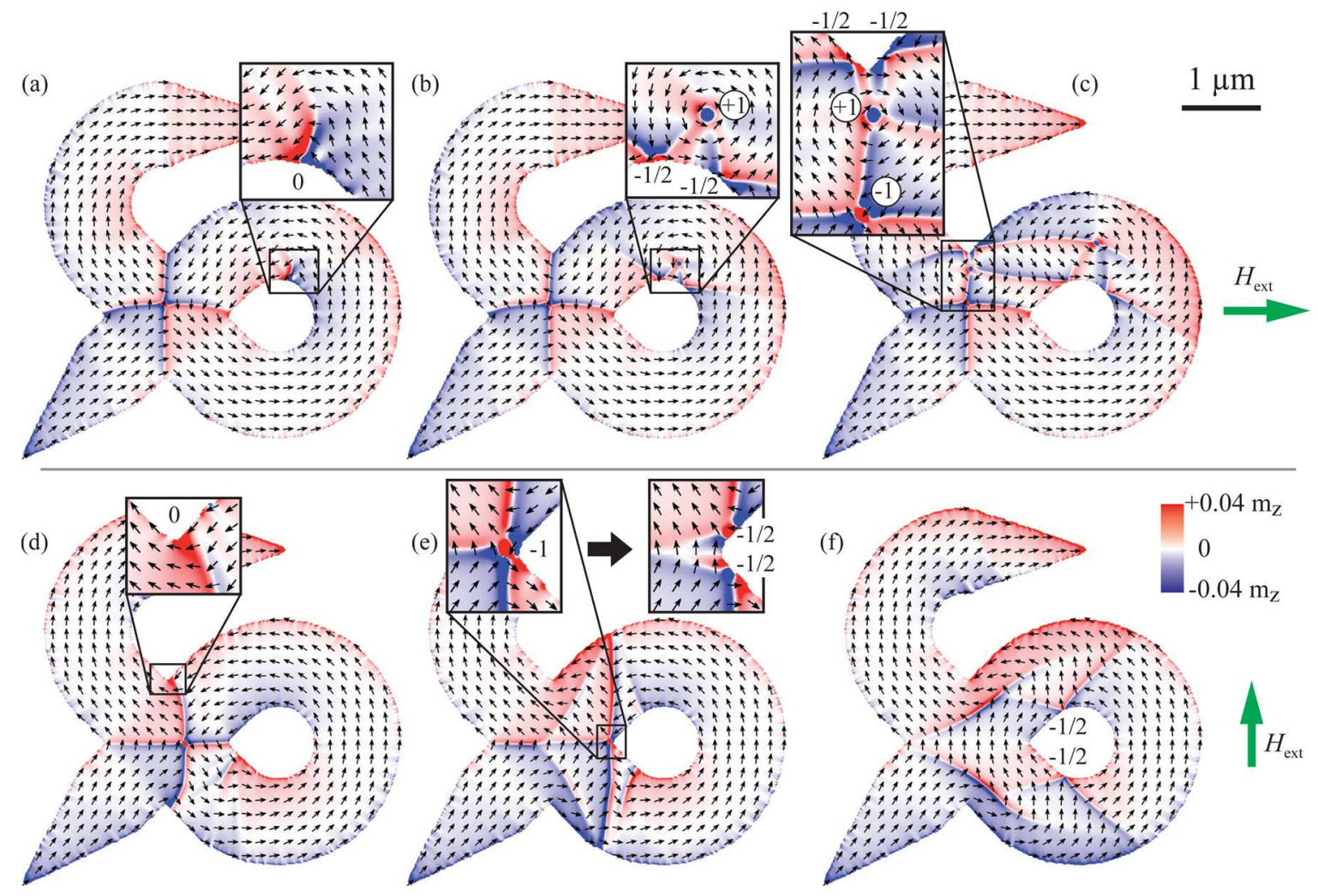

FIG. 6. (a)-(c) Sequence of the simulated annihilation process of the antivortex with an orientation of $c=-1$ by a static external field of $\mu_{0} H_{\text {ext }}=11 \mathrm{mT}$ in $x$-direction. (a) A $180^{\circ}$ domain wall forms in a curved segment of the $\varphi$-shaped structure. (b) A vortex nucleates from the domain wall. (c) The upper $90^{\circ}$ domain wall of the antivortex detaches from the corner of the wire junction forming another vortex. This vortex moves to the center of the junction and both the vortex and the antivortex are annihilated. (d)-(f) Sequence of the annihilation of the antivortex at $\mu_{0} H_{\text {ext }}=14 \mathrm{mT}$ in $y$-direction. (d) The upper and lower $90^{\circ}$ domain walls are no longer pinned at the respective corner and start moving into the arms. (e) The antivortex moves to the right corner and is annihilated there. Two edge defects are formed that move further into the wire. The numbers denote the winding number of the respective magnetization pattern. 
nucleates and a domain that no longer points antiparallel to the external field is created, see Fig. 6(b). This tilts the magnetization in the upper right arm and destabilizes the upper $90^{\circ}$ domain wall of the antivortex. It detaches from the corner of the junction and another vortex nucleates from the former domain wall. The new vortex moves towards the antivortex and both are annihilated, see Fig. 6(c). This process is labeled $\mathrm{V}_{\text {arm }}$ in Fig. 5(b) since the annihilation of the antivortex starts with the creation of a vortex in a wire arm. The second process, labeled $\mathrm{V}_{\mathrm{DW}}$, is similar, but a $90^{\circ}$ domain wall of the antivortex detaches from the corner of the junction via a vortex nucleation without a preceding vortex nucleation in a curvature of the structure. The last process, $\mathrm{AV}_{\mathrm{an}}$, is only observed for a bias field in $y$-direction. The upper and lower domain walls of the antivortex depin from the corners, but are still attached to the boundary of the structure, see Fig. 6(d). The domain walls and the antivortex start moving to the right until the antivortex core reaches the right corner of the wire junction, see Fig. 6(e). Here, the antivortex is annihilated and two edge defects ${ }^{27}$ form, which move further along the inner boundary of the curved wire. Similarly, edge defects are generated, when a vortex nucleates in the other processes described above. The magnetic texture of these edge defects can be described by half integer winding numbers $n= \pm 1 / 2$. When taking all magnetic defects that are generated or annihilated during the annihilation of the antivortex into account, it can be observed that the sum of the winding number of the whole structure remains $n_{\text {sum }}=-1$, the same as the initial antivortex, cf. Fig. 6 . This holds for all bias field angles up to the maximum field strength of $15 \mathrm{mT}$ in the simulation. The different annihilation processes exhibit no special symmetry that can be found in the $\varphi$-shaped structure, cf. Figs. 5(b) and 5(d), but give a possible explanation of the anisotropy of the measured annihilation fields. For most cases, the antivortex is not pushed to the boundary of the wire junction and destroyed there, but its magnetic texture is distorted by changes of the magnetization far from the antivortex. The annihilation processes for the real element may differ from the ones observed in the simulations, however, a possible diversity in the annihilation mechanism is revealed. Thus in the absorption and magnetoresistance measurements, it cannot be distinguished, if the annihilation field of the antivortex is measured, or a field at which the magnetization at some point in the $\varphi$-shaped structure is reversed by the external field as in the $V_{\text {arm }}$ process.

The annihilation fields determined by MR measurements and micromagnetic simulations for a static antivortex are in good agreement, but the results from absorption spectroscopy, where the antivortex is gyrating at the resonance frequency exhibit much smaller fields, see Fig. 5(a). This discrepancy may not be attributed to high gyration radii and an expulsion of the antivortex from the decentered equilibrium position at a certain bias field. An estimation of the gyration radius $r_{\text {gyr }}$ can be done by results from transmission X-ray microscopy on antivortices in elements with comparable dimensions. ${ }^{7,9}$ In these works, maximal gyration radii of $95 \mathrm{~nm}$ to $115 \mathrm{~nm}$ measured at $140 \mathrm{MHz}$ and $120 \mathrm{MHz}$, respectively, are measured before core switching starts. Assuming a similar switching threshold for the here presented structures and a linear dependence of the gyration radius on the excitation field $H_{\mathrm{hf}}$, the radius $r_{\mathrm{gyr}}$ can be estimated to be about $10 \mathrm{~nm}$, a tenth of the maximal radius. Core switching is reached at $\mu_{0} H_{\mathrm{hf}}=3.0 \mathrm{mT}$, cf. Fig 3(a), and $0.3 \mathrm{mT}$ are used as the excitation field strength for the measurements of the bias field dependence on the resonance frequency, cf. Fig. 4(a). The displacement for the lowest annihilation field for the absorption measurements with $\mu_{0} H_{\text {an }}\left(180^{\circ}\right)=1.4 \mathrm{mT}$ can be deduced from the simulations, where the constant deflection rate yields a displacement of the equilibrium position for this field of about $20 \mathrm{~nm}$. The maximal displacement for a gyrating antivortex at this position can thus be estimated to be under $30 \mathrm{~nm}$ away from the center of the wire junction, whereas it can be deflected up to $142 \mathrm{~nm}$ in the static case. Consequently, the reduced annihilation fields for the deflection of an excited antivortex cannot be attributed to the gyration radius of the antivortex, but rather to a reduction of the activation fields of the above described processes of domain wall depinning and vortex nucleation by the high frequency field. A similar reduction of the switching field of a nanoparticle by radio-frequency field pulses is described by Thirion et al. in Ref. 28. However, the maximal gyration radius of about $100 \mathrm{~nm}$ derived from the transmission X-ray microscopy results ${ }^{7,9}$ before core switching starts and the minimal deflection distance of $142 \mathrm{~nm}$ for the static case obtained by micromagnetic simulations could explain the drastic decrease in the absorption signal for increasing excitation fields in the core switching regime, cf. Fig. 3(a). It supports the above mentioned hypothesis of an antivortex destruction for strong excitation field strength and high gyration radii. $^{29}$

Another comparison of the antivortex and the vortex can be made concerning the critical velocity needed for core switching. For vortices, a critical switching velocity $v_{\text {crit }}=2 \pi r f_{\text {res }}$ was found to be $320 \mathrm{~m} / \mathrm{s}$ by analytical and micromagnetic calculations 5 for vortices in discs or $250 \mathrm{~m} / \mathrm{s}$ for vortices in squares by absorption measurements. ${ }^{13,23}$ The squares have comparable dimensions as the wire width and thickness of the structures used to stabilize the antivortex. The vortices in these squares exhibit a resonance frequency of $320 \mathrm{MHz}$ and thus reach a gyration radius of about $124 \mathrm{~nm}$ at the critical velocity. The microscopy results for antivortices yield a much lower critical velocity of about $85 \mathrm{~m} / \mathrm{s}$ at the comparable radii of $95 \mathrm{~nm}$ to $115 \mathrm{~nm}$. The cause of the low critical velocity needs to be investigated and if the critical velocity is also applicable as a universal criterion for the switching process of antivortices.

\section{CONCLUSIONS}

The gyrotropic eigenmode of isolated antivortices has been measured by high frequency absorption spectroscopy for varying excitation field strengths in the linear, non-linear, and core switching regimes. The behavior of isolated antivortices is similar to one of the excited vortices. When comparing antivortices in our structures with vortices in squares of similar dimensions, the gyrotropic mode of the antivortex has a lower resonance frequency. To induce core switching, the antivortex needs to be exposed to a much stronger excitation field than the vortex.

Deviations of the confining harmonic potential of the antivortex due to a static in-plane field have been 
demonstrated by the shift of the resonance frequency in the absorption measurements.

To complement the anisotropy of the annihilation fields found in the absorption spectroscopy, magneto-resistance measurements as well as micromagnetic simulations have been performed. While the annihilation fields determined from the MR measurements and the micromagnetic simulations without an excitation of the antivortex match quite well, the annihilation fields drop about a half for the absorption spectroscopy, where the antivortex is excited. Moreover, a much more distinct anisotropy of the annihilation can be found for the excited state. It is demonstrated by means of micromagnetic simulations that for some field angles, the annihilation of the antivortex is caused by a rotation of the magnetization within an arm of the structure far away from the antivortex itself, thus destabilizing the antivortex.

These results show that a simple quasi particle description for the antivortex is no longer applicable for the case of a strong excitation and a deflection of the equilibrium position by a bias field exceeding about $5 \mathrm{mT}$ for our structures, but the change of the whole magnetization of the $\varphi$-shaped structure has to be taken into account.

In order to find an expansion for the confining harmonic potential of isolated antivortices, further investigations are needed. A direct depiction of the magnetization by transmission X-ray microscopy can show the deformation of the circular trajectory of the excited antivortex depending on the bias field and the annihilation process. The influence of the $\varphi$-shaped structure on the antivortex may also be investigated by increasing the length of the straight wire junction and the radius of the curved wire segments.

\section{ACKNOWLEDGMENTS}

We thank Ulrich Merkt for discussions, encouragement, and continuous support and Michael Volkmann for superb technical assistance. Financial support by the Deutsche Forschungsgemeinschaft via Sonderforschungsbereich 668 is gratefully acknowledged.

${ }^{1}$ S.-K. Kim, K.-S. Lee, Y.-S. Yu, and Y.-S. Choi, Appl. Phys. Lett. 92, 022509 (2008).

${ }^{2}$ S. Bohlens, B. Krüger, A. Drews, M. Bolte, G. Meier, and D. Pfannkuche, Appl. Phys. Lett. 93, 142508 (2008).

${ }^{3}$ A. Drews, B. Krüger, G. Meier, S. Bohlens, L. Bocklage, T. Matsuyama, and M. Bolte, Appl. Phys. Lett. 94, 062504 (2009).
${ }^{4}$ B. Van Waeyenberge, A. Puzic, H. Stoll, K. Chou, T. Tyliszczak, R. Hertel, M. Fähnle, H. Brückl, K. Rott, G. Reiss, I. Neudecker, D. Weiss, C. H. Back, and G. Schütz, Nature 444, 461 (2006).

${ }^{5}$ K. Y. Guslienko, K.-S. Lee, and S.-K. Kim, Phys. Rev. Lett. 100, 027203 (2008).

${ }^{6}$ T. Kamionka, M. Martens, A. Drews, B. Krüger, O. Albrecht, and G. Meier, Phys. Rev. B 83, 224424 (2011).

${ }^{7}$ T. Kamionka, M. Martens, K. W. Chou, A. Drews, T. Tyliszczak, H. Stoll, B. Van Waeyenberge, and G. Meier, Phys. Rev. B 83, 224422 (2011).

${ }^{8}$ K.-S. Lee, K. Y. Guslienko, J.-Y. Lee, and S.-K. Kim, Phys. Rev. B 76, 174410 (2007).

${ }^{9}$ T. Kamionka, M. Martens, K. W. Chou, M. Curcic, A. Drews, G. Schütz, T. Tyliszczak, H. Stoll, B. Van Waeyenberge, and G. Meier, Phys. Rev. Lett. 105, 137204 (2010).

${ }^{10}$ H. Wang and C. E. Campbell, Phys. Rev. B 76, 220407 (2007).

${ }^{11}$ M. Kammerer, H. Stoll, M. Noske, M. Sproll, M. Weigand, C. Illg, G. Woltersdorf, M. Fähnle, C. Back, and G. Schütz, Phys. Rev. B 86, 134426 (2012).

${ }^{12}$ R. Hertel, S. Gliga, M. Fähnle, and C. M. Schneider, Phys. Rev. Lett. 98, 117201 (2007).

${ }^{13}$ A. Vansteenkiste, K. W. Chou, M. Weigand, M. Curcic, V. Sackmann, H. Stoll, T. Tyliszczak, G. Woltersdorf, C. H. Back, G. Schütz, and B. Van Waeyenberge, Nat. Phys. 5, 332 (2009).

${ }^{14}$ T. Shinjo, T. Okuno, R. Hassdorf, K. Shigeto, and T. Ono, Science 289, 930 (2000).

${ }^{15}$ K. J. Kirk, S. McVitie, J. N. Chapman, and C. D. W. Wilkinson, J. Appl. Phys. 89, 7174 (2001).

${ }^{16}$ K. Shigeto, T. Okuno, K. Mibu, T. Shinjo, and T. Ono, Appl. Phys. Lett. 80, 4190 (2002)

${ }^{17}$ M. Pues, M. Martens, T. Kamionka, and G. Meier, Appl. Phys. Lett. 100, 162404 (2012).

${ }^{18}$ I. Horcas, R. Fernández, J. M. Gómez-Rodríguez, J. Colchero, J. GómezHerrero, and A. M. Baro, Rev. Sci. Instrum. 78, 013705 (2007).

${ }^{19}$ A. Drews, B. Krüger, G. Selke, T. Kamionka, A. Vogel, M. Martens, U. Merkt, D. Möller, and G. Meier, Phys. Rev. B 85, 144417 (2012).

${ }^{20}$ B. Krüger, A. Drews, M. Bolte, U. Merkt, D. Pfannkuche, and G. Meier, J. Appl. Phys. 103, 07A501 (2008).

${ }^{21}$ T. J. Silva, C. S. Lee, T. M. Crawford, and C. T. Rogers, J. Appl. Phys. 85, 7849 (1999).

${ }^{22}$ The data have been post processed by a median filter to eliminate outliners.

${ }^{23}$ M. Martens, T. Kamionka, M. Weigand, H. Stoll, T. Tyliszczak, and G. Meier, Phys. Rev. B 87, 054426 (2013).

${ }^{24}$ H. H. Langner, T. Kamionka, M. Martens, M. Weigand, C. F. Adolff, U. Merkt, and G. Meier, Phys. Rev. B 85, 174436 (2012).

${ }^{25}$ M. J. Donahue and D. G. Porter, "OOMMF User's Guide, Version 1.0," Interagency Report NISTIR 6376, National Institute of Standards and Technology, Gaithersburg 1999.

${ }^{26}$ J.-S. Kim, O. Boulle, S. Verstoep, L. Heyne, J. Rhensius, M. Kläui, L. J. Heyderman, F. Kronast, R. Mattheis, C. Ulysse, and G. Faini, Phys. Rev. B 82, 104427 (2010).

${ }^{27}$ O. Tchernyshyov and G.-W. Chern, Phys. Rev. Lett. 95, 197204 (2005).

${ }^{28}$ C. Thirion, W. Wernsdorfer, and D. Mailly, Nature Mater. 2, 524 (2003).

${ }^{29}$ In the transmission X-ray microscopy investigations in Refs. 9 and 7, we occasionally observed the disappearance of the antivortex core after several switching processes. 